\title{
Could Neuromuscular Electrical Stimulation Really Lead to Changes in Voice of Swallowing Disorder Caused by a Stroke?
}

\author{
Haewon Byeon ${ }^{1}$ and Sunghyoun $\mathrm{Cho}^{2 *}$ \\ ${ }^{1}$ Dept. of Speech Language Pathology \& Audiology, Nambu University, Gwangju, \\ South Korea, \\ ${ }^{2 *}$ Dept. of Physical Therapy, Nambu University, Gwangju, South Korea, \\ 'byeon@nambu.ac.kr, ${ }^{2}$ geriatricpt1@naver.com
}

\begin{abstract}
This study investigated the effect of Neuromuscular Electrical Stimulation (NMES) on the loudness, pitch and quality of the voice of the males diagnosed with dysphagia by stroke. NMES was conducted on experimental group of 15 patients for 2 months repeatedly while compensatory strategies for dysphagia were performed on control group of 15 patients. Loudness, pitch $\left(F_{o}\right)$ and quality (Jitter, Shimmer, NNE; Normalized Noise Energy) of the voice was measured by using acoustic-phonetic analysis. For statistical test, difference in voice change after the treatments was compared by using analysis of covariance (ANCOVA). As the result of analysis, NMES and compensatory strategies had significant difference in intensity, jitter and shimmer $(p<.05)$ while they did not in pitch and NNE. After the intervention of NMES, voice loudness of the subjects was enhanced and periodicity of vocal cord vibration (Jitter, Shimmer) was stabilized. The results of this study imply that NMES may have effect on enhancement of voice loudness and stabilization of periodicity of vocal cord vibration.
\end{abstract}

Keywords: Dysphagia, Vital Stim, Stroke, Voice, Acoustic Analysis

\section{Introduction}

Swallowed food bolus goes through 3 stages of oral cavity, pharynx and esophagus and if a problem arises in these swallowing stages, dysphagia takes place. Especially, prevalence rate of dysphagia is very high in stroke patients with abnormal nerve or muscle involved in swallowing function [1]. In the case of acute stroke patients, the report of occurrence rate of dysphagia after stroke varies from $37 \%$ to $45 \%$ [2-5] and, in the case of early acute stroke patients, aspiration which is passage of food into respiratory tract and penetration which is the remaining of food in the larynx can take place [4]. In particular, silent aspiration occurs in approximately $40 \%$ of dysphagia patient from stroke [6] and thus causes high possibility of outbreak of aspiration pneumonia, which makes early diagnosis and intervention necessary [7]. For treatments of patients with oral cavity-pharynx dysphagia from stroke, compensatory strategies such as change of posture, change in amount and speed of food and temperature-tactile stimulation method have traditionally been practiced and, in the case of chronic functional disorder of specific swallowing muscle, surgical interventions such as cricopharyngeal myotomy are sometimes utilized $[1,8]$.

Recently, Neuromuscular Electrical Stimulation (NMES) is practiced in the swallowing rehabilitation of stroke patients and there have been reports that it has significant rehabilitation effect [9-13]. Studies comparing effects of traditional dysphagia treatment with those of NMES also reported that there were no significant differences of treatment effects in the randomized controlled trials to which blinding tests were applied [14] and, in a retrospective cohort study, 
Blumenfeld, et al., [15] reported that NMES enhanced swallowing function more significantly than traditional treatments. These preceding studies explained its mechanism as NMES emitting delicate electric current on the skin and thus raising external muscles of larynx and lifting capacity of larynx, rehabilitating swallowing function $[10,16]$.

Meanwhile, Freed and Wijting reported that NMES not only has positive effect on the rehabilitation of swallowing function but changes loudness and pitch of the voice $[17,18]$. Production of voice is explained by Source-Filter theory composed of formation and resonance process of sound energy [19]. Voice which is sound energy is produced by vocal fold vibration, when internal muscles of the larynx take charge of the vocal fold movement. In the case of stroke patients, however, abnormal voice is produced with its pitch, loudness and quality deviated from normal range due to paralysis of internal muscles of larynx caused by damage of central or peripheral nerve.

In the studies on the effect of electric stimulation on laryngeal muscles, different results have been reported depending on whether the position of the electric stimulation was muscles outside of larynx or internal laryngeal muscles. Experimental studies which directly implemented electric stimulation on internal laryngeal muscles reported that electric stimulation has effect on the function of vocal fold [17-18]. According to a study by Zealear, et al., [20], electric stimulation on internal laryngeal muscle of the patients with bilateral vocal fold paralysis maintained vocal fold abduction by vitalizing the function of posterior cricoarytenoid muscle. In addition, according to Bidus, et al., [21], stimulation on vocal cord adductor on the patients with abductor spasmodic dysphonia significantly increased syllable duration and voiceless consonant duration. However, studies which implemented electric stimulation on the neck skin did not find any significant change in vocal fold function and voice [16, 18].

Although the relationship between Neuromuscular Electrical Stimulation (NMES) and the voice has not been clearly verified, there have been reports on cases of change in loudness of dysphagia patients' voice in the process of NMES [18]. In addition, preceding studies on the effect of NMES on voice [17, 18] only analyzed the result of voice change by temporary electric stimulation on normal people and there is lack of voice study on repetitive NMES on patients with dysphagia. As the theoretical ground of NMES lies in the principle of neuroplasticity which is reorganization or realignment of damaged function in the surrounding areas [17], there is a limitation to confirm change of voice by NMES with a response to single stimulation.

This study investigated the effect of repeated NMES on the loudness, pitch and quality of voice of the dysphagia patients from stroke by using acoustic-phonetic analysis.

\section{Methods}

\subsection{Subjects}

The study subjects were 30 dysphagia patients hospitalized from August 2011 through October 2012 in rehabilitation hospital located in Incheon due to stroke. Among these 30 patients, 15 patients in experimental group received NMES intervention while 15 patients in contrast group received compensatory strategies. Selection criteria for dysphagia patients were as follows; first, sub-acute patients not exceeding 6 months after the diagnosis of dysphagia; second, those without history of lung disease, laryngeal disease and disease in oral speech mechanism; third, those able to make phonation; fourth, males over the age of 50 through less 
than 70; fifth, those with over the score of 20 as the result of Mini-Mental State Examination - Korean version (MMSE-K) [22], which is translated to be able to understand the examination method; sixth, those without experience of receiving voice therapy and NMES before the date of study.

\subsection{Material}

\section{1) VitalStim}

For the treatment of dysphagia, NMES treatment equipment VitalStim (Chattanooga Group, Tennessee, USA) [17] was used which was approved by Food \& Drug Administration (Figure 1). This study used 2-channel alternating current and frequency of vibration was $80 \mathrm{~Hz}$ with stimulation width of $300 \mathrm{usec}$. Stimulation per cycle was set at 700usec and the intensity of the electric current started from $6.5 \mathrm{~mA}$ and was raised by $0.5 \mathrm{~mA}$ each time within the range in which patients can endure the inconvenience and pain.

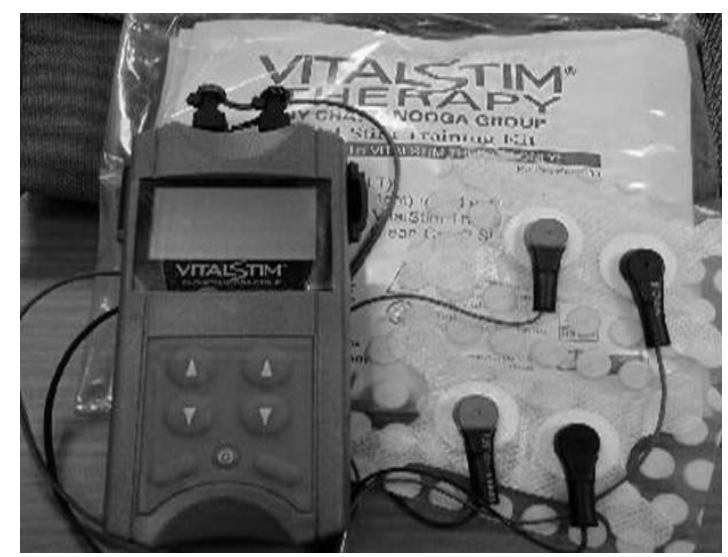

Figure 1. VitalStim

\section{2) Vocal Assessment for Voice}

For measurement of Loudness, pitch, and quality of the voice, Vocal Assessment for Voice (Version 4.5, Tiger Drs, Inc, USA) [23] were used (Figure 2). As analysis indexes for voice measurement, this study used mean-intensity for loudness, mean $\mathrm{F}_{0}$ for pitch and, for quality, jitter, shimmer and NNE (Normalized Noise Energy) respectively (Figure 3).

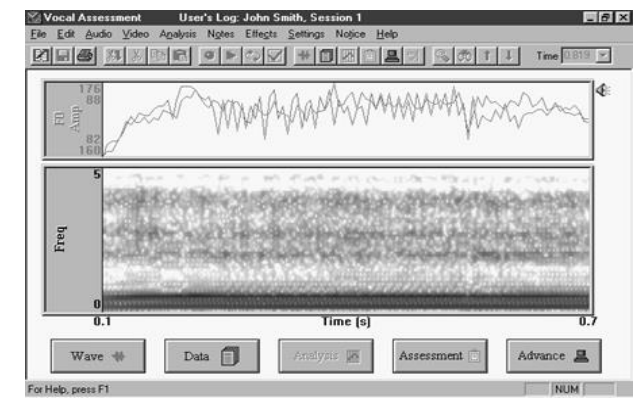

Figure 2. Vocal Assessment for voice 


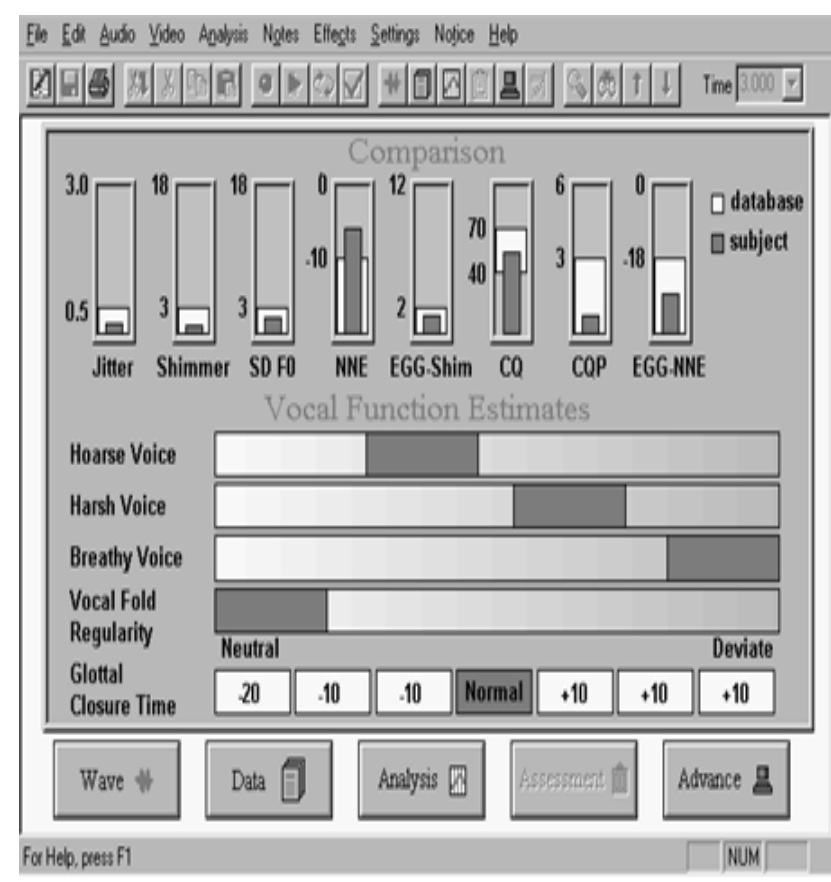

Figure 3. Parameter of Vocal Assessment

\subsection{Measurement}

\section{1) Neuromuscular Electrical Stimulation}

NMES was conducted in the same time period using 2-channel electric stimulant for 25 minutes per session until the completion of the study and all the subjects received the treatment 5 times a week except weekends for 2 months, which is 40 times in total. Referring to the study of Humbert, et al., [16], two electrodes were attached on superior to the hyoid bone and superior to the thyroid notch respectively (Figure 4).

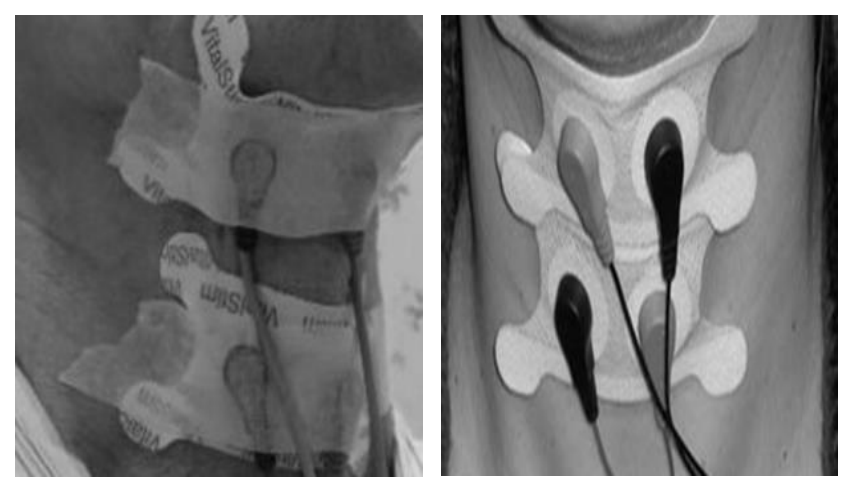

Figure 4. Electrode Placement of VitalStim

\section{2) Compensatory Strategies}

For compensatory strategies, chin tuck, head rotation, Mendelsohn maneuver, supraglottic swallow technique, super-supraglottic swallow technique and effortful swallow were used [1].

\section{3) Voice Analysis}

Voice analysis was performed in the method of directly recording subjects' prolonged phonation of vowels in acoustic analysis program in a noiseless voice test room within 1 hour after NMES intervention. Subjects did not receive any rehabilitation treatment after 
NMES intervention until the voice analysis during the study period in order to control the effect of subjects fatigue from other rehabilitation treatments on voice and to minimize the variability of voice by conducting voice analysis at the same time zone. PG48-LC microphone of Shure Corp. was used and the distance from the microphone and lips were maintained at $5 \mathrm{~cm}$ for measurement under identical condition.

Voice recording was conducted in the method of making prolonged phonation of vowel /e/. All the voice recordings were one phonation data and stable section was designated in the vowel phonation by comparing oscillograph and time point of fundamental frequency, after which pitch and quality was analyzed.

\subsection{Statistical Analysis}

For general characteristics of subjects in the baseline stage, mean and standard deviation using technical analysis were presented and difference between groups was analyzed by using independent sample t-test. In addition, treatment was defined as explanatory variable while voice loudness, pitch and quality (Jitter, Shimmer, NNE) were defined as an outcome and baseline was defined as covariance, and difference of change in outcome was compared after the treatment using one-way analysis of covariance (ANCOVA). Statistical test was analyzed using IBM SPSS version 22.0(IBM Inc., Chicago, Illinois).

\section{Results}

\subsection{General Characteristics of the Subjects}

General characteristics of the subjects are presented in Table 1. Average age of the subjects was 60.0, number of years of education was 10.2 and MMSE score was 22.5. Pitch was $132.3 \mathrm{~Hz}$, loudness was $42.0 \mathrm{~dB}$, and for quality, Jitter was $1.1 \mathrm{~dB}$ $(\%)$ and Shimmer was $3.3 \mathrm{~dB}(\%)$ while NNE was $-5.5 \mathrm{~dB}(\%)$, which all belong to abnormal range compared with normal standards suggested in Vocal Assessment for Voice.

As the result of independent sample t-test conducted to find out the difference between groups in baseline stage, it turned out there was significant difference only in education level $(\mathrm{p}<.05)$. Average number of education years of NMES group was 11.3, bigger than compensatory strategies group's 9.2.

Table 1. General Characteristics of Subjects in the Baseline Stage, Mean \pm SD

\begin{tabular}{lrrrr}
\hline Variables & NMES & $\begin{array}{c}\text { Compensatory } \\
\text { strategies }\end{array}$ & Total & $\mathrm{p}$ \\
\hline \hline Age & $61.5 \pm 5.2$ & $59.3 \pm 3.6$ & $60.0 \pm 4.5$ & 0.186 \\
Years of education & $11.3 \pm 2.8$ & $9.2 \pm 1.3$ & $10.2 \pm 2.4$ & 0.016 \\
Post of Trauma (month) & $4.1 \pm 1.1$ & $3.8 \pm 0.8$ & $3.9 \pm 0.9$ & 0.370 \\
MMSE-K & $22.8 \pm 1.9$ & $22.3 \pm 0.9$ & $22.5 \pm 1.5$ & 0.411 \\
F $_{0}(\mathrm{~Hz})$ & $130.8 \pm 9.0$ & $133.9 \pm 8.7$ & $132.3 \pm 8.9$ & 0.347 \\
Intensity $(\mathrm{dB})$ & $41.8 \pm 3.6$ & $42.2 \pm 3.1$ & $42.0 \pm 3.3$ & 0.677 \\
Jitter $(\%)$ & $1.1 \pm 0.3$ & $1.1 \pm 0.2$ & $1.1 \pm 0.2$ & 0.810 \\
Shimmer $(\%)$ & $3.4 \pm 0.9$ & $3.3 \pm 0.6$ & $3.3 \pm 0.8$ & 0.733 \\
NNE $(\%)$ & $-5.7 \pm 3.3$ & $-5.3 \pm 2.6$ & $-5.5 \pm 2.9$ & 0.708 \\
\hline
\end{tabular}


In order to investigate the effect of NMES on loudness, pitch and quality, a total of 30 subjects were evenly divided and placed in NMES and compensatory strategies with 15 subjects in each group respectively, and pretest and posttest were performed in sequence (Table 1). First, as the result of analysis of interaction effect between pretest and treatment to see if the basic assumption of ANCOVA is satisfied, the slopes of regression line of pretest were identical in two groups as interaction effect was not statistically significant. In addition, Levene test on homogeneity of variance also confirmed that variances are the same.

As the result of one-way ANCOVA which controlled pretest with covariance, NMES and compensatory strategies have significant difference in intensity, jitter and shimmer $(\mathrm{p}<.05)$. As the result of estimation of parameter, NMES significantly raised intensity by $2.0 \mathrm{~dB}$ higher than compensatory strategies on average while it significantly lowered jitter and shimmer by $0.3 \%$ respectively. Especially, in the case of NMES group, jitter declined from average $1.1 \mathrm{~dB}(\%)$ in pretest to average $0.5 \mathrm{~dB}(\%)$ in posttest $(\mathrm{p}<.01)$, reaching within $0.5 \mathrm{~dB}(\%)$ which is normal in the standard of Vocal Assessment for Voice, and shimmer declined from average 3.4 $\mathrm{dB}(\%)$ in pretest to average $2.5 \mathrm{~dB}(\%)$ in posttest $(\mathrm{p}<.01)$, reaching normal standard of within $3.0 \mathrm{~dB}(\%)$.

Table 2. Results of Acoustic Evaluations after Neuromuscular Electrical Stimulation

\begin{tabular}{lcccccc}
\hline \multirow{2}{*}{ Variables } & \multicolumn{2}{c}{ NMES } & \multicolumn{2}{c}{ Compensatory strategies } & $\mathrm{F}$ & $\mathrm{p}$ \\
& Pre-treatment & Post-treatment & Pre-treatment & Post-treatment & & \\
\hline \hline $\mathrm{F}_{0}(\mathrm{~Hz})$ & $130.8 \pm 9.0$ & $131.8 \pm 10.8$ & $133.9 \pm 8.7$ & $134.2 \pm 8.9$ & 0.001 & 0.935 \\
Intensity (dB) & $41.8 \pm 3.6$ & $44.5 \pm 3.3$ & $42.2 \pm 3.1$ & $42.5 \pm 3.0$ & 9.313 & 0.005 \\
Jitter $(\%)$ & $1.1 \pm 0.3$ & $0.5 \pm 0.2$ & $1.1 \pm 0.2$ & $0.8 \pm 0.2$ & 14.661 & 0.001 \\
Shimmer $(\%)$ & $3.4 \pm 1.0$ & $2.5 \pm 0.6$ & $3.3 \pm 0.6$ & $2.8 \pm 0.7$ & 4.358 & 0.046 \\
$\mathrm{NNE}(\%)$ & $-5.7 \pm 3.3$ & $-6.7 \pm 2.3$ & $-5.3 \pm 2.6$ & $-6.8 \pm 2.3$ & 1.293 & 0.266 \\
\hline
\end{tabular}

\section{Discussion}

This study investigated the effect of Neuromuscular Electrical Stimulation (NMES) on loudness, pitch and quality of voice in males with dysphagia caused by stroke. The findings of this study are as follows:

First, there was no significant change in pitch. Cricothyroid muscle is responsible for change in pitch and Vilkman, et al., [24] said that cricothyroid muscle works independently of the effect of external laryngeal muscles. Therefore, NMES is presumed to have no significant effect on the change in pitch in this study.

Second, loudness of voice significantly increased by approximately $4 \mathrm{~dB}$ on average, which is different from the result of Fowler, et al., [18] that NMES did not have significant effect on the change of voice loudness. This difference is deemed to be caused by the fact that the study of Fowler, et al., [18] performed NMES only once on the normal people. As the NMES is based on the principle of neuroplasticity which is reorganization or realignment of damaged function in the surrounding areas [17] in the process of repetitive stimulation, there is limitation to confirm change of voice by NMES with a response to single stimulation. In concluding that there was no statistical significance of NMES since directions of increase or decrease of loudness were all different although temporary NMES did cause changes in individual subjects' voice loudness, Fowler, et al., [18] suggested possibility of physiological change by repeated stimulation of neck muscles. In 
spite of reports by numerous studies that NMES had significant effect only on external laryngeal muscles $[10,16]$, the possibility of NMES having minute effect on vitalization of internal laryngeal muscles cannot be excluded [16]. Therefore, in order to verify this possibility, it is necessary for future studies to measure the movement of internal laryngeal muscles together with acoustic-phonetic analysis.

Third, in quality, both jitters which represent frequency perturbation of vibration of vocal fold and shimmer which represents amplitude perturbation reached normal range. Stabilization of jitter and shimmer by NMES in this study is presumed to be caused by enhancement of larynx lifting function or recovery of muscle function which controls vibration of vocal fold [25]. However, as periodicity of vocal cord vibration can be affected by the change in pressure of the lungs [26], aerodynamic test in addition to acoustic-phonetic test is required so that complex factors related to frequency change in vibration of vocal fold can be investigated in follow-up studies.

Fourth, although Normalized Noise Energy (NNE) significantly increased by NMES intervention, it still did not reach normal level. NNE which is a figure of total vocal energy less harmonic signal energy is effectively used in distinguishing voice with pathological problems from normal voice [27, 28]. If a gap develops in vocal fold due to vocal fold paralysis and other reasons, turbulent noise can be created which increases noise in voice with more air leaking [29, 30]. Even though there was significant increase in NNE figures during the course of NMES, they were still in pathologically abnormal range. Therefore, in order for follow-up studies to verify increase of NNE by NMES based on this study, intervention period of more than 2 months will be required.

The limitations of this study are as follows; first, it is difficult to generalize the result of the study due to limited number of subjects. As many patients with acute dysphagia receive tracheostomy, there was difficulty to recruit patients with dysphagia who were able to make a large number of phonation during study period. Second, there is a limitation in explaining the causal relationship between NMES intervention and rehabilitation of muscles affecting voice only with acousticphonetic analysis results. In order to prove the causal relationship between NMES intervention and rehabilitation of internal laryngeal muscles, it is necessary to confirm the degree of muscle recovery by using laryngeal electromyography or neck tomography. In order to find out the relationship between NMES and voiceproducing muscles in more detail, future studies are required to conduct acoustic phonetic analysis and neuromuscular test to investigate the relationship.

\section{Conclusion}

Subjects' voice loudness increased after NMES intervention and periodicity of vocal cord vibration (Jitter, Shimmer) was also stabilized. The result of this study implies that NMES intervention may have effect on the enhancement of voice loudness and stabilization of periodicity of vocal cord vibration.

\section{References}

[1] J. A. Logemann, "The evaluation and treatment of swallowing disorders", Current Opinion in Otolaryngology \& Head and Neck Surgery, vol. 6, (1998), pp. 395-400.

[2] D. J. Ramsey, D. G. Smithard and L. Kalra, "Early assessments of dysphagia and aspiration risk in acute stroke patients", Stroke, vol. 34, (2003), pp. 1252-1257.

[3] D. T. Wade and R. L. Hewer, "Motor loss and swallowing difficulty after stroke: frequency, recovery, and prognosis", Acta Neurologica Scandinavica, vol. 76, (1987), 50-54.

[4] D. Kidd, J. Lawson, R. Nesbitt and J. MacMahon "Aspiration in acute stroke: A clinical study with video fluoroscopy”, QJ Med., vol. 86, (1993), pp. 825-829.

[5] I. R. Odderson, J. C. Keaton and B. S. McKenna, "Swallow management in patients on an acute stroke pathway: quality is cost effective”, Arch Phys Med Rehabil., vol. 76, (1995), pp. 1130-1133. 
[6] D. Ramsey, D. Smithard and L. Kalra, "Silent aspiration: what do we know?", Dysphagia, vol. 20, (2005), pp. 218-225.

[7] P. E. Marik, "Aspiration pneumonitis and aspiration pneumonia", New England Journal of Medicine, vol. 344, (2001), pp. 665-671.

[8] N. De Zoysa, P. Patel, A. Trinidade, P. V. Vlastarakos, P. Kothari and G. Mochloulis, "Success of cricopharyngeal myotomy for specific and non-specific cervical dysphagia", B-ENT, vol. 9, (2013), pp. 307-312.

[9] M. L. Freed, L. Freed, R. L. Chatburn and M. Christian, "Electrical stimulation for swallowing disorders caused by stroke", Respiratory Care, vol. 46, (2001), pp. 466-474.

[10] V. Leelamanit, C. Limsakul and A. Grater, "Synchronized electrical stimulation in treating pharyngeal dysphagia”, Laryngoscope, vol. 112, (2002), pp. 2204-2210.

[11] M. Kiger, C. S. Brown and L. Watkins, "Dysphagia management: an analysis of patient outcomes using VitalStim therapy compared to traditional swallow therapy", Dysphagia, vol. 21, (2006), pp. 243-253.

[12] S. J. Shin, J. M. Park, D. U. Lee, M. E. Jung, E. Y. Yu and M. K. Yu, "The effects of electrical stimulation on the neck muscles of patients with a swallowing disorder caused by a stroke", The Journal of Korean Academy of Occupational therapy, vol. 17, (2009), pp. 17-28.

[13] G. Y. Shaw, P. R. Sechtem, J. Searl, K. Keller, T. A. Rawi and E. Dowdy, "Transcutaneous neuromuscular electrical stimulation (VitalStim) curative therapy for severe dysphagia: myth or reality?", Annals of Otology, Rhinology \& Laryngology, vol. 116, (2007), pp. 36-44.

[14] M. Bulow, R. Speyer, L. Baijens, V. Woisard and O. Ekberg, "Neuromuscular electrical stimulation (NMES) in stroke patients with oral and pharyngeal dysfunction", Dysphagia, vol. 23, (2008), pp. 302309.

[15] L. Blumenfeld, Y. Hahn, A. Lepage, R. Leonard and P. C. Belafsky, "Transcutaneous electrical stimulation versus traditional dysphagia therapy: a non concurrent cohort study", Otolaryngol Head Neck Surg, vol. 135, (2006), pp. 754-757.

[16] I. A. Humbert, C. J. Poletto, K. G. Saxon, P. R. Kearney and C. L. Ludlow, "The effect of surface electrical stimulation on vocal fold position", Laryngoscope, vol. 118, (2008), pp. 14-19.

[17] M. L. Freed and Y. Wijting, "Training manual for patient assessment and treatment using VitalStim electrical stimulation", TN: Chattanooga Group, (2003).

[18] L. P. Fowler, M. Gorham-Rowan and E. R. Hapner, "An exploratory study of voice change associated with healthy speakers after Transcutaneous Electrical Stimulation to laryngeal muscles", Journal of Voice. E-pub, (2010).

[19] G. Fant, "Acoustic theory of speech production", The Hague: Mounton, (1968).

[20] D. L. Zealear, C. R. Billante, M. S. Courey, G. D. Sant Anna and J. L. Netterville, "Electrically stimulated glottal opening combined with adductor muscle botox blockade restores both ventilation and voice in a patient with bilateral laryngeal paralysis", Ann Otol Rhinol Laryngol, vol. 111, (2002), pp. $500-506$.

[21] K. A. Bidus, G. R. Thomas and C. L. Ludlow, "Effects of adductor muscle stimulation on speech in abductor spasmodic dysphonia", Laryngoscope, vol. 110, (2000), pp. 1943-1949.

[22] J. Park and Y. Kwon, "Standardization of Korean version of the mini-mental state examination (MMSE-K) for use in the elderly", Part $\Pi$, diagnostic validity, Journal of the Korean Neuropsychiatric Association., vol. 28, (1989), pp. 508-513.

[23] D. Z. Huang, E. Engr and S. B. Engr, "Vocal assessment user's manual", version 4.5, WA: Tigers DRS, Inc., (1996).

[24] E. Vilkman, A. Sonninen, P. Hurme and P. Korkko, "External laryngeal frame function in voice production revisited: a review", Journal of Voice, vol. 10, (1996), pp. 78-92.

[25] C. T. Ferrand, "Speech Science: An integrated approach to theory and clinical practice (2nd ed)", MA: Allyn and Bacon, (2006).

[26] I. R. Titze, "Principles of voice production", Englewood Cliffs, NJ: Prentice Hall, (1994).

[27] H. S. Kasuya, K. Ogawa and E. Mashima, "Normalized noise energy as an acoustic measure to evaluate pathological voice", J Acoust Soc Amer., vol. 80, (1986), pp. 1329-1334.

[28] K. Y. Lim, M. S. Shin, J. B. An and O. L. Jung, "The study of correlation between HNR and NNE", Korean Journal of Speech Sciences, vol. 8, (2001), pp. 235-241.

[29] J. P. H. Pabon and R. Plomp, "Automatic phonetogram recording supplemented with acoustical voice quality parameters", J Speech Hear Res., vol. 31, (1988), pp. 710-722.

[30] H. Byeon and S. Cho, "Voice change after neuromuscular electrical stimulation to laryngeal muscles", Advanced Science and Technology, vol. 105, (2015), pp. 28-31. 


\section{Authors}

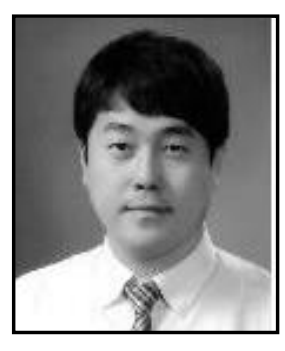

Haewon Byeon, received the DrSc degree in Biomedical Science from Ajou University School of Medicine. He is a professor in Department of Speech Language Pathology \& Audiology and director of Speech Language Pathology Center in Nambu University. His recent interests focus on health promotion and biostatistics.

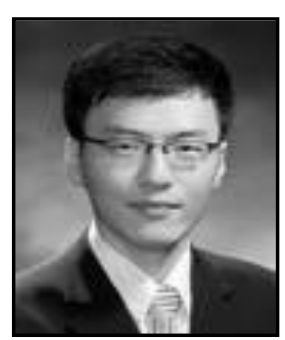

Sunghyoun Cho, received the $\mathrm{PhD}$ degree in Physical Therapy from Daegu University. He is a professor in Department of Physical Therapy in Nambu University, Gwangju, Republic of Korea. His recent interests focus on Biomechanics and Therapeutic exercise. 
International Journal of Bio-Science and Bio-Technology

Vol.7, No.6 (2015) 